\title{
Mathematical model of multi-frequency piezoresonance oscillation system
}

\author{
Alexander A. Zelensky ${ }^{1}$, Sergey K. Pidchenko ${ }^{2}$, Alla A. Taranchuk ${ }^{2}$ \\ ${ }^{1}$ N.E. Zhukovsky National Aerospace University «KhAI», Kharkov, Ukraine \\ ${ }^{2}$ Khmelnitskiy National University, Khmelnitskiy, Ukraine
}

\section{Email address:}

azelens@mail.ru (A. A. Zelensky), sergpchn@yandex.ru (S. k. Pidchenko), allatr@ukr.net (A. A. Taranchuk)

\section{To cite this article:}

Alexander A. Zelensky, Sergey K. Pidchenko, Alla A. Taranchuk. Mathematical Model of Multi-Frequency Piezoresonance Oscillation System. Communications. Vol. 1, No. 1, 2013,pp. 1-8. doi: 10.11648/j.com.20130101.11

\begin{abstract}
The paper represents the basic model of multi-frequency piezoresonance oscillation system (MPOS) - the piezoresonance devices (PRD) core, which enables to study the processes of establishing multi-frequency oscillation mode and its stability. The basic structure of multi-channel multi-frequency PRD core, which is based on principles of filter schemes, is proposed, and the main designations are entered. The peculiarities of truncated differential equations for amplitude, phase and auto-bias voltage of MPOS for the quantity of simultaneously generated frequencies $m \geq 2$ are examined. On the example of three-frequency mode of oscillation under polynomial approximation of transferable characteristics of active elements the characteristic cases of establishing oscillations in MPOS are represented. The area of a steady three-frequency oscillating behavior is defined and the assessment of time of establishment of oscillations and value of group runout of frequencies is made. Received results enable to form a new approach to construction of piezoresonance devices with controlled dynamics, which are represented in the form of adaptive controlled systems with predictive standard model and develop on its basis the new class of invariant to destabilizing disturbing PRD factors. On the basis of such approach there is the principle of using natural redundancy in multi-frequency basis of PRD core - multi-frequency oscillation system, which enables not only to synthesize the system with current identification of disturbing factors on basis of instruments of invariance theory, but also do the adaptation of PRD in accordance with their influences.
\end{abstract}

Keywords: Stabilization of Oscillations, Multi-Frequency Quartz Oscillatory System, Invariant Piezoresonance System

\section{Introduction}

Anisotropic properties of crystalline piezoelements cause different types of elastic ties of mechanical stresses and deformations, which lead to appearance of unwanted resonances in piezoresonance devices (PRD). Usually multifrequency of crystalline piezoelements, taking into account destabilizing factors (DF), which have an effect on PRD, is considered as undesirable effect, which is tried to be removed by means of structural and technological methods, which is often at variance with demands of mass production.

On the basis of conducted research [1-4] a new approach has been formed to provide the invariance of piezoresonance devices. Such approach is based on the conception of PRD as a dynamic object with natural redundancy in the frequensy basis (multi- frequency) and current identification of reversible on PRD the destabilizing factors. At the same time, quartz resonator $(\mathrm{QR})$ has frequency - determining (stabilizing) function and the additional measuring function, which enables to accomplish continuous identification of DF.

Magnitude estimation of destabilizing disturbing factors are determined by special algorithmic processing of multi-frequency signals, which defined such approach as multi-frequency - algorithmic $[2,4]$. These valuations of DF can be used for solving the tasks of compensation, statting or as a result of solving individual task of synchronous measuring of several physical quantities in local volume of measurement field [8]. The theoretic basis of multi- frequency algorithmic approach is the fundamental works on the theory of invariance and its use in the field of measuring technique and automatic devices [3]. The material basis of multi-frequency - algorithmic approach is the rapid development of microelectronic and microprocessor technique, the use of which in the complex with multi- frequency excitation of $\mathrm{QR}$, is the "revolutionary tendency" in precision piezolelectronics [2]. 
Recently the development of multi-frequency - algorithmic methods of provision the invariance of PRD led to their use in several two- frequency (two- mode) piezoresonance oscillators and measurement systems with current identification of temperature influence. However, the research in such field [5-15] do not take into account the whole spectrum of specific conditions of modern PRD functioning, which reveals in sharp changes in temperature rate and substantial vibration and mechanical effects, which makes the task of providing the invariance in the conditions of parametrical non-stationarity essentially difficult. At that the following mechanisms, which define the dynamics of multifrequency PRD, can be defined [16,17]:

1. The conversion to multi-frequency oscillation mode (MOM) of QR caused by competition of oscillations in the PRD excitation channels reduces the oscillation stability and complicates considerably the character of transient processes, which leads to extending the time for setting MOM as compared to one- frequency mode [18];

2. The use of MOM under the conditions of temperature DF requires firmer controlling of summary power of excitation and the thermal QR condition. The presence of rapidly changing thermal flows, the considerable temperature gradients leads to irregular heating of QR piezoelement and causes specific thermo dynamic shift of QR frequency, which distorts sharply its temperature and frequency characteristics, which altogether reduces the long-term stability of PRD oscillations substantially $[19,20]$;

3. Though quartz resonator is inert to temperature DF, the inertial properties are negligible towards mechanical forces (such as impacts, vibration, acoustic noises). The effect of vibration and mechanical DF causes parasite frequency modulation of PRD oscillation (the decrease of short-term stability), which especially develops in frequency measuring transducers where $\mathrm{QR}$ with enchanted force sensitivity is used [21].

Listed above factors cause the necessity of conducting further research on expanding functional potential of multifrequency algorithmic methods, which are based on introducing PRD as dynamic object. This requires the development of generic model of multi-frequency PRD for studying the dynamics on the stage of oscillation determination as well as under the conditions of temperature and vibration influence for multi-frequency oscillation mode with the quantity of generated frequency $m \geq 2$.

Present work introduces the basic model of multifrequency piezoresonance oscillatory system (MPOS) PRD core, which allows to analyze the processes of establishing multi-frequency oscillation mode and its stability. The second section proposes the basic structure of multi-channel multi-frequency PRD core and introduces new indications. In the third section features of an outputs of the truncated differential equations are considered for amplitudes, phases and auto-bias voltages of MPOS for the quantity of simultaneously generated frequency $m \geq 2$.

The fourth section represents characteristic cases of establishing oscillations in MPOS on the example of three-frequency oscillation mode under polynomial approximation of transferable characteristics of active elements. The field of stable three-frequency oscillation mode is defined, and the estimation of the time of establishing the oscillations and value of group frequency overshoot is done. Further the discussion of the most considerable received results is given.

\section{Multi-frequency Core Structure of a Piezoresonance Oscillation System}

High effectiveness of multi-frequency generating mode is provided by condition of high stability in generated oscillations. This is possible only with using filtering schemes in which QR is embedded in feedback circuit and is excited in the frequencies close to those of its own consequent resonances. At limited quantity of generated oscillations the other oscillator schemes can be used, for example those of oscillating type [22].

The basic core architecture represents MPOS to have principles of creating filtering schemes implemented (fig. 1). It incorporates passive multi-frequency quartz quadripole unit (MQU) on the base of quartz resonator with $\mathrm{m}$ - frequencies generating $z_{q_{j}}$ and n excitation channels embedding the generalized non-linear component (NLC) and phasing selective feedback circuit (FBC). The automatic bias circuits with complex equivalent resistance $z_{b_{i}}$ are used for stabilizing NLCi operational mode. The selective non-linear circuits $\mathrm{FBC}$ with gain $K_{j i}\left(j w, u_{\Sigma}, \tau\right), j=\overline{1, m}$, $i=\overline{1, n}$, except for their function to set required amplitude-phase ratio in excitation channels, provide significant reducing competition in oscillations due to their own selective properties $K_{j i}(j w)$ and also automatic adjustment $K_{j i}\left(u_{\Sigma}\right)$ of oscillation amplitudes for fixing the specified (ultimately acceptable) power dissipation on QR.

Figure 1 has the following symbols' identifications:

$z_{\mathrm{in}_{\Sigma}}=R_{\mathrm{in}_{\Sigma}} /\left(1+j w \tau_{\mathrm{in}_{\Sigma}}\right), R_{\mathrm{in}_{\Sigma}}^{-1}=\sum_{i} R_{\mathrm{in}_{i}}^{-1}, C_{\mathrm{in}_{\Sigma}}=\sum_{i} C_{\mathrm{in}_{i}}-$ the complex equivalent total resistance of partial FC input circuits;

$$
\begin{aligned}
& z_{\text {out }_{\Sigma}}=R_{\text {out }_{\Sigma}} /\left(1+j w \tau_{\text {out }_{\Sigma}}\right), R_{\text {out }_{\Sigma}}^{-1}=\sum_{i} R_{\text {out }_{i}}^{-1}, \\
& C_{\text {out }_{\Sigma}}=\sum_{i} C_{\text {out }_{i}}-\text { the complex equivalent total resistance }
\end{aligned}
$$
of output NLCi circuits;

$z_{\mathrm{ab}_{i}}=R_{\mathrm{ab}_{i}} /\left(1+j w \tau_{\mathrm{ab}_{i}}\right)-$ complex resistance of auto-bias circuit i;

$$
\tau_{\mathrm{ab}_{i}}=R_{\mathrm{ab}_{i}} C_{\mathrm{ab}_{i}}, \quad \tau_{\mathrm{in}_{\Sigma}}=R_{\mathrm{in}_{\Sigma}} C_{\mathrm{in}_{\Sigma}}, \quad \tau_{\text {out }_{\Sigma}}=R_{\text {out }_{\Sigma}} C_{\text {out }_{\Sigma}}-
$$
time constants;

$$
i_{\Sigma}=\sum_{i} i_{\text {out }}\left(e_{i}\right)-\text { total current of NLCi; }
$$


$e_{i}(\tau)=\sum_{j} v_{i}(\tau)+E_{i}+\widetilde{E}_{i}(\tau)$ - control voltage in $\mathrm{NLC}_{\mathrm{i}}$ input, where $E_{i}, \widetilde{E}_{i}(\tau)$ - constant and variable components of auto-bias voltage;

$$
u_{\Sigma}(\tau)=U_{0}(\tau)+\sum_{j} U_{j}(\tau) \cdot \cos \left[w_{j} t+\varphi_{j}(\tau)\right] \quad \text { - the total }
$$

voltage in FC circuit input, where $U_{j}(\tau), w_{j}$ and $\varphi_{j}(\tau)$ envelope, frequency and phase of oscillation $\mathrm{j}$ correspondently, $\tau=t-t_{0}-$ time interval from initial moment $t_{0}$ (MPOS startup moment) [16].

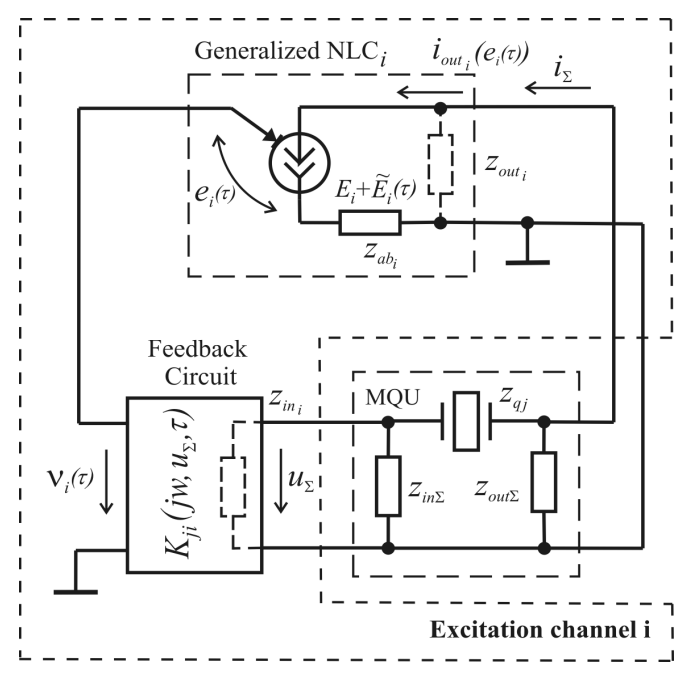

Figure 1. The basic core architecture.

\section{Truncated Differential Equations for Amplitudes and Phases of Oscillations}

MPOS operation (fig. 1) is described by the system of differential equations:

$$
\begin{gathered}
u_{\Sigma}=Z(p) \cdot \sum_{i} i_{\text {out }_{i}}\left(\sum_{j} K_{j i} \cdot u_{\Sigma}, \widetilde{E}_{i}, E_{i}\right) \\
E_{i}=-z_{\mathrm{ab}_{i}}(p) \cdot i_{\mathrm{out}_{i}}\left(\sum_{j} K_{j i} \cdot u_{\Sigma}, \widetilde{E}_{i}, E_{i}\right) \\
Z(p)=\left(z_{\mathrm{in}_{\Sigma}}(p) z_{\text {out }_{\Sigma}}(p)\right) /\left(z_{\mathrm{in}_{\Sigma}}(p)+z_{\text {out }}(p)+z_{q}(p)\right)
\end{gathered}
$$

- symbolic gain of MQU, representing control resistance of MPOS; $p \equiv d / d t$.

Representing control resistance $Z(p)$ (3) as the ratio

$$
Z(p)=\frac{\delta \cdot P(p, \delta)}{Q(p, \delta)},
$$

where $P(p, \delta), Q(p, \delta)$ - polynomials of $p ; \delta$ - low parameter, physically determined for resonance systems as damping ratio, and taking into account that in the general case solving system (1) is as sum of oscillations with frequencies close to resonance ones of $\mathrm{QR} \omega_{q_{j}}$

$$
u_{\Sigma}=\sum_{j=1}^{m} U_{j}(\tau) \cos \left(\omega_{q_{j}} t+\varphi_{j}(\tau)\right)
$$

where $U_{j}(\tau)$ and $\varphi_{j}(\tau)$ - slowly changing parameters, the output current of $\mathrm{NLC}_{i}$ with low parameter $\delta$ accuracy can be written as:

$$
\begin{gathered}
i_{\text {out }_{i}}\left(e_{i}\right)=I_{o_{i}}\left(V_{j i}, E_{i}, \widetilde{E}_{i}\right)+ \\
+\sum_{j=1}^{m} I_{j i}\left(V_{j i}, E_{i}, \widetilde{E}_{i}\right) \cos \left[\omega_{q_{j}} t+\psi_{j i}(t)\right],
\end{gathered}
$$

where $I_{o i}-$ constant component; $I_{j i}=\bar{S}_{j i}\left(V_{j i}, E_{i}, \widetilde{E}_{i}\right) \cdot V_{j i}=$ $=\bar{S}_{j i}\left(V_{j i}, E_{i}, \widetilde{E}_{i}\right) \cdot U_{j} \cdot K_{j i} ; \psi_{j i}=\varphi_{j}+\Delta \varphi_{j i}$;

$K_{j i}, \Delta \varphi_{j i}$ - gain and phase shift of circuit $i$ of FBC for resonance frequency $j$ of $\mathrm{QR}$;

$$
\bar{S}_{j i}\left(V_{j i}, E_{i}, \tilde{E}_{i}\right)=\frac{1}{V_{j i}} L\left[i_{\text {out }_{i}}\left(V_{j i}, E_{i}, \tilde{E}_{i}\right)\right]-\text { mean slope for }
$$

oscillation $j ; \underset{j}{L}[\bullet]-$ mean operator.

Having entered complex amplitudes $\dot{U}_{j}=U_{j} \exp \left(\varphi_{j}\right)$, $\dot{I}_{j i}=I_{j i} \exp \left(j \psi_{j i}\right)$ and complex $\mathrm{FBC}$ gain $\dot{K}_{j i}=K_{j i} \exp \left(j \Delta \varphi_{j i}\right)$, and split real and imaginary units the truncated equations for amplitudes, MPOS oscillation phases and auto-bias voltages become $[23,24]$ :

$$
\begin{aligned}
& T_{j} \frac{d U_{j}}{d t}=\left[R_{\mathrm{e}_{j}} K_{\phi_{j}} \sum_{i=1}^{n} \bar{S}_{j i}\left(V_{j i}, \widetilde{E}_{i}, E_{i}\right) \times\right. \\
& \left.\times K_{j i} \cos \left(\Delta \varphi_{j i}+\Delta_{\phi_{j}}\right)-1\right] \cdot U_{j} ; \\
& T_{j} \frac{d \varphi_{j}}{d t}=R_{\mathrm{e}_{j}} K_{\phi_{j}} \sum_{i=1}^{n} \bar{S}_{j i}\left(V_{j i}, \widetilde{E}_{i}, E_{i}\right) \times \\
& \times K_{j i} \sin \left(\Delta \varphi_{j i}+\Delta_{\phi_{j}}\right)-\Delta \omega_{j} T_{j}
\end{aligned}
$$

$$
T_{\mathrm{ab}_{i}} \frac{d \widetilde{E}}{d t}=-\left[R_{\mathrm{ab}_{i}} I_{0 i}\left(V_{j i}, \widetilde{E}_{i}, E_{i}\right)+\widetilde{E}_{i}\right] ; j=\overline{1, m} ; i=\overline{1, n},
$$

or as generalized matrix

$$
\begin{aligned}
& \mathbf{T} \frac{d \mathbf{U}}{d t}=\left[\mathbf{G}_{\mathbf{R}} \cdot \mathbf{R}-\mathbf{E}_{(\mathrm{mm})}\right] \cdot \mathbf{U} ; \\
& \mathbf{T} \frac{d \mathbf{\Phi}}{d t}=\left[\mathbf{G}_{\mathbf{I}} \cdot \mathbf{R}-\Delta\right] \cdot \mathbf{E}_{(\mathrm{ml})} ;
\end{aligned}
$$




$$
\mathbf{T}_{\mathbf{a b}} \frac{d \widetilde{\mathbf{E}}}{d t}=-\left(\mathbf{R}_{\mathbf{a b}} \cdot \mathbf{I}_{\mathbf{0}}+\widetilde{\mathbf{E}}\right)
$$

where $\mathbf{T}=\operatorname{diag}\left(T_{1}, \ldots, T_{m}\right), T_{\mathbf{a b}}=\operatorname{diag}\left(\tau_{\mathrm{ab}_{1}}, \ldots, \tau_{\mathrm{ab}_{n}}\right)$ - time constant matrixes of partial oscillation system $T_{j}=2 / \omega_{q_{j}} \delta_{j}$ of $m \times m$ dimensions and auto-bias circuits NLCi of $n \times n$ dimensions; $\mathbf{U}=\left(U_{1}, \ldots, U_{m}\right)^{\mathrm{T}}$, $\boldsymbol{\Phi}=\left(\varphi_{1}, \ldots, \varphi_{m}\right)^{\mathrm{T}}$ and $\widetilde{\mathbf{E}}=\left(\widetilde{E}_{1}, \ldots, \widetilde{E}_{n}\right)^{\mathrm{T}}-$ vectors of oscillation amplitudes and phases of $m$ dimension and vector of auto-bias voltage of $n$ dimension;

$$
\mathbf{G}_{\mathbf{R}}=\operatorname{Re} \dot{\mathbf{G}}=|\dot{\mathbf{G}}| \cos \Delta \varphi^{\mathrm{T}}, \mathbf{G}_{\mathbf{I}}=\operatorname{Im} \dot{\mathbf{G}}=|\dot{\mathbf{G}}| \sin \Delta \varphi^{\mathrm{T}}
$$

- matrixes of real and imaginary units of equivalent complex conductance of active part of MPOS $|\dot{\mathbf{G}}|=\left(\bar{S}_{j i} K_{j i}\right)_{j=1, i=1}^{m, n}$ of $m \times n$ dimensions;

$R=R_{\mathrm{e}} \cdot K_{\varphi} \quad-\quad$ matrix of reduced resistances; $R_{\mathrm{e}}=\operatorname{diag}\left(R_{\mathrm{e}_{1}}, \ldots, R_{\mathrm{e}_{m}}\right)-$ matrix of equivalent resistances $R_{\mathrm{e}_{j}}=R_{\mathrm{in}_{\Sigma}} \cdot R_{\text {out }_{\Sigma}} /\left(R_{\mathrm{in}_{\Sigma}}+R_{\text {out }_{\Sigma}}+R_{q_{j}}\right) \quad$ of $m \times m$ dimensions, $\mathbf{K}_{\phi}=\operatorname{diag}\left(K_{\phi_{1}}, \ldots, K_{\phi_{m}}\right)-$ matrix of equivalent phase link gains $K_{\phi_{j}}=1 / \sqrt{1+\left(\omega_{q_{j}} \cdot T_{\phi_{j}}\right)^{2}}$, $T_{\phi_{j}} \approx R_{\mathrm{in}_{\Sigma}}\left(C_{0}+C_{\mathrm{in}_{\Sigma}}\right)+R_{\text {out }_{\Sigma}}\left(C_{0}+C_{\text {out }_{\Sigma}}\right)$ - time constant of equivalent phase link, providing phase shift $\Delta \varphi_{\phi_{j}}=-\operatorname{arctg}\left(\omega_{q_{j}} \cdot T_{\phi_{j}}\right)$ between immediate values of voltage $u(t)$ and current $i(t) ; \omega_{q_{j}}, C_{0}$ - resonance frequency and parallel capacity of $\mathrm{QR}$;

$$
\Delta \varphi=\left(\Delta \varphi_{j i}+\Delta \varphi_{\phi_{j}}\right)_{j=1, i=1}^{m, n} \text { - matrix of } m \times n \text { dimen- }
$$

sions, which provides phase relations in excitation channels of MPOS;

$\Delta=\operatorname{diag}\left(\Delta \omega_{1} T_{1}, \ldots, \Delta \omega_{m} T_{m}\right)-$ matrix of generalized detuning, $\quad \Delta \omega_{j}=\omega_{q_{j}}^{2}\left(R_{\mathrm{in}_{\Sigma}} \tau_{\mathrm{in}_{\Sigma}}+R_{\mathrm{out}_{\Sigma}} \tau_{\text {out }_{\Sigma}}\right) / 2 Q_{q_{j}} R_{q_{j}}$ - frequency adjustment to oscillation $\mathrm{j}$ explained by the fact that reduction was produced relative to natural frequencies of QR $\omega_{q_{j}}$ with no respect to phase-shift in FC circuits; $Q_{q_{j}}, R_{q_{j}}$ - quality factor and dynamic resistance of $\mathrm{QR}$ for oscillation frequency $\mathrm{j}$;

$R_{\mathrm{ab}}=\operatorname{diag}\left(R_{\mathrm{ab}_{1}}, \ldots, R_{\mathrm{ab}_{n}}\right)-$ matrix of auto-bias resistance of $n \times n$ dimensions;

$$
\mathbf{I}_{\mathbf{0}}=\left(I_{0_{1}}, \ldots, I_{0_{n}}\right)^{\mathrm{T}}-\text { vector of constant components of }
$$
output currents $i_{\text {ouț }}\left(e_{i}\right)$;

$E_{(\mathbf{m m})}, E_{(\mathbf{m} \mathbf{l})}-$ unit matrix of $m \times m$ dimensions and unit column vector $m \times 1$; $m$ - quantity of generating frequencies, $n$ - quantity of excitation channels.

\section{Dynamics of Multi-Frequency Piezo- resonance Oscillation System}

The case considers three-frequency excitation of the main mode of oscillations of multi-frequency QR and two additional ones - informative: temperature and vibration $(m=n=3$ ) $[25,26]$. At polynomial approximation the output current of $\mathrm{NLC}_{\mathrm{i}}$ (fig. 1) can be represented as

$$
i_{\text {out }_{i}}\left(e_{i}\right)=I_{s}\left[\widehat{a}_{0}+\sum_{r=1}^{k} \widehat{a}_{r}\left(\widehat{v}_{i}+X_{\mathrm{b}_{i}}\right)^{r}\right], i=\overline{1, n}
$$

where $\hat{v}_{i}=v_{i} \cdot S_{0} / I_{s}-$ normalized control voltage; $X_{\mathrm{b}_{i}}=\bar{x}_{\mathrm{b}_{i}}+\widetilde{x}_{\mathrm{b}_{i}}, \bar{x}_{\mathrm{b}_{i}}=\left(E_{i}-E_{s}\right) \cdot S_{0} / I_{s}, \widetilde{x}_{\mathrm{b}_{i}}=\widetilde{E}_{i} \cdot S_{0} / I_{s}-$ constant and variable components of normalized bias; $\hat{a}_{0}=a_{0} / I_{s}, \quad \hat{a}_{r}=a_{r} \cdot I_{s}^{(r-1)} / S_{0}^{r}-$ normalized coefficients of approximating polynomial; $I_{s}, E_{s}-$ the coordinates of the point in the center of current-voltage characteristic (CVC) with maximal gain slope $S_{0} ; k=3,5$.

Having used trigonometry formulas of multiple argument and averaging accordantly to (6), the spectral components in NLCi input at approximation by polynomial of the third degree will be written as

$$
\begin{aligned}
I_{j i} & =V_{j i} \cdot \bar{S}_{j i}=V_{j i} \cdot S_{0}\left[A_{1 i}+0,75 \cdot \widehat{a}_{3} B_{1 i}\right] \\
I_{0_{i}} & =I_{s}\left[A_{2 i}+0.5\left(\widehat{a}_{2}+3 \widehat{a}_{3} X_{\mathrm{b}_{i}}\right) B_{2 i}\right] .
\end{aligned}
$$

Having substituted the expressions (14), (15) into truncated equations $(10)-(12)$ and assuming $K_{\phi_{j}} \approx 1$ and $\Delta \varphi_{\phi_{j}} \approx 0$, the three-frequency MPOS $(m=3)$ motion equation with three non-linear components $(n=3)$ will be obtained:

$$
\begin{gathered}
\xi_{j} \frac{d \hat{U}}{d \hat{t}}=S_{0} R_{\mathrm{e}_{j}} K_{j j} \cdot \hat{U}_{j} \times \\
\times\left(\sum_{i=1}^{3}\left(A_{1 i}+0.75 \hat{a}_{3} B_{1 i}\right) \cdot \gamma_{j i}^{2} \frac{K_{i i}}{K_{j j}}-\frac{1}{S_{0} R_{\mathrm{e}_{j}} K_{j j}}\right) \\
\xi_{j} \frac{d \varphi_{j}}{d \hat{t}}=S_{0} R_{\mathrm{e}_{j}} K_{j j} \cdot \sum_{i=1}^{3}\left(A_{1 i}+0.75 \hat{a}_{3} B_{1 i}\right) \times \\
\times \gamma_{j i} \sqrt{1-\gamma_{j i}^{2}} \frac{K_{i i}}{K_{j j}}-\xi_{j} \Delta \omega_{j}
\end{gathered}
$$




$$
\begin{gathered}
\mu_{i} \frac{d X_{\mathrm{b}_{i}}}{d \tilde{t}}=-S_{0} R_{\mathrm{ab}_{i}} \times \\
\times\left(A_{2 i}+\frac{X_{b_{i}}}{S_{0} R_{\mathrm{ab}_{i}}}+0,5\left(\underline{a}_{2}+3 \underline{a}_{3} X_{b_{i}}\right) B_{2 i}\right)+\bar{x}_{b_{i}} .
\end{gathered}
$$

The correspondent equations (16) - (18) at approximating transfer characteristics by polynomial of the fifth degree (13) are given in [16].

The following symbols used in expressions (14) - (18) stand for:

$$
\begin{gathered}
A_{1 i}=\sum_{r=1}^{3} r \widehat{a}_{r} \cdot X_{\mathrm{b}_{i}}^{(r-1)}, A_{2 i}=\widehat{a}_{0}+\sum_{r=1}^{3} \widehat{a}_{r} \cdot X_{\mathrm{b}_{i}}^{r}, \\
B_{1 i}=K_{i i}^{2} \sum_{k=1}^{3} b_{k i j} \cdot \hat{U}_{k}^{2}, B_{2 i}=K_{i i}^{2} \sum_{j=1}^{3} \gamma_{j i}^{2} \cdot \widehat{U}_{j}^{2} ; \\
b_{k i j}=\gamma_{k i}^{2}, \text { for } k=j \text { and } b_{k i j}=2 \gamma_{k i}^{2}, \text { for } k \neq j ; \\
\gamma_{j i}=\frac{K_{j i}}{K_{i i}}=\cos \Delta \varphi_{j i}-\text { coefficients of suppressing in- }
\end{gathered}
$$
ter-channel interference (parasite) oscillations $(j \neq i)$; $\widehat{U}_{j}=S_{0} U_{j} / I_{s}-$ normalized voltage in MQU output; $\xi_{j}=T_{j} / \max _{1 \leq j \leq 3} T_{j}, \quad \mu_{i}=T_{\mathrm{ab}_{i}} / \max _{1 \leq j \leq 3} T_{j}-$ normalized time constants of partial oscillation system $\mathrm{j}$ and bias circuit $\mathrm{i}$; $\bar{t}=t / \max _{1 \leq j \leq 3} T_{j}-$ normalized time; $j=\overline{1,3}, i=\overline{1,3}$.

Activity of excited oscillations is determined by regeneration parameter to be found from (16) when $\widehat{U}_{j}=0$ :

$$
\begin{gathered}
K_{j}^{r e g}=R_{\mathrm{e}_{j}} K_{j j} S_{\mathrm{s}_{j}}= \\
=R_{\mathrm{e}_{j}} K_{j j} S_{0} \times \sum_{i=1}^{3}\left(\sum_{r=1}^{3,5} r \widehat{a}_{r} X_{\mathrm{s}_{j}}^{(r-1)}\right) \gamma_{j i} \frac{K_{i i}}{K_{j j}},
\end{gathered}
$$

where $S_{\mathrm{s}_{j}}$ - gain slope of volt-amps diagram in still point for oscillation $j ; X_{\mathrm{s}_{j}}$ - bias in the still point for $\mathrm{NLC}_{i}$, being the solution for non-linear equation:

$$
S_{0} \cdot R_{\mathrm{ab}_{i}}\left(\hat{a}_{0}+\frac{X_{\mathrm{b}_{i}}}{S_{0} \cdot R_{\mathrm{ab}_{i}}}+\sum_{r=1}^{3} \hat{a}_{r} \cdot X_{\mathrm{b}_{i}}^{r}\right)-\bar{x}_{\mathrm{b}_{i}}=0
$$

Figures 2 and 3 demonstrate distinguished case of stabilizing oscillations in three-frequency MPOS, as result of numerical integrating equations (16) - (18) in MATLAB system. The bipolar transistors (2SC9018) are used as active components (NLCi), which have average statistical values of CVC parameters: $S_{0}=0.1 \mathrm{~A} / \mathrm{B} \quad ; \quad \hat{a}_{0}=0.95$; $\hat{a}_{1}=0.55 ; \hat{a}_{2}=0.051 ; \hat{a}_{3}=-0.054$, when approximation error $\varepsilon \leq 5 \%$. Initial values of low-varying parameters made: $T_{j}=0.1 \mathrm{sec} ; \quad T_{\mathrm{ab}_{i}}=1 \cdot 10^{-4} \mathrm{sec} ; \quad R_{\mathrm{ab}_{i}}=1 \mathrm{k} \Omega$; $K_{j j}=-20 \mathrm{~dB} ; \quad \bar{x}_{\mathrm{b}_{i}}=30$.

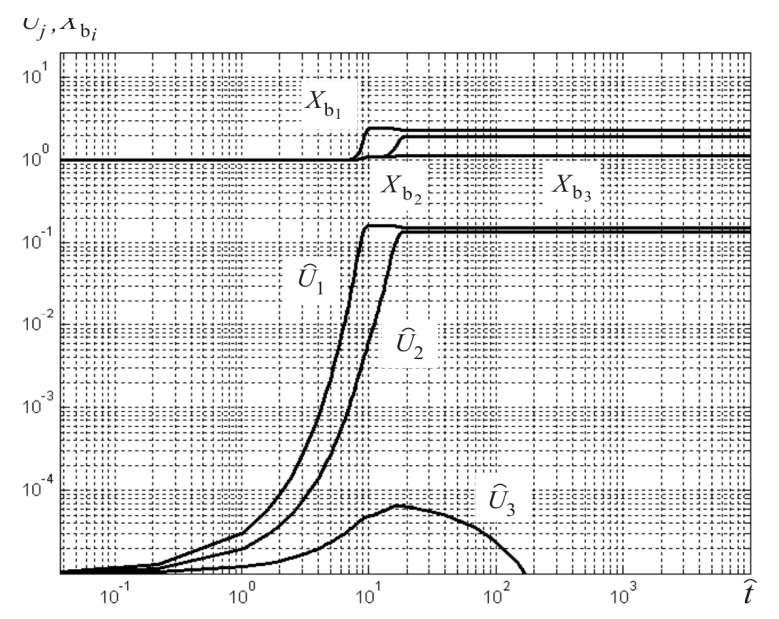

(a)

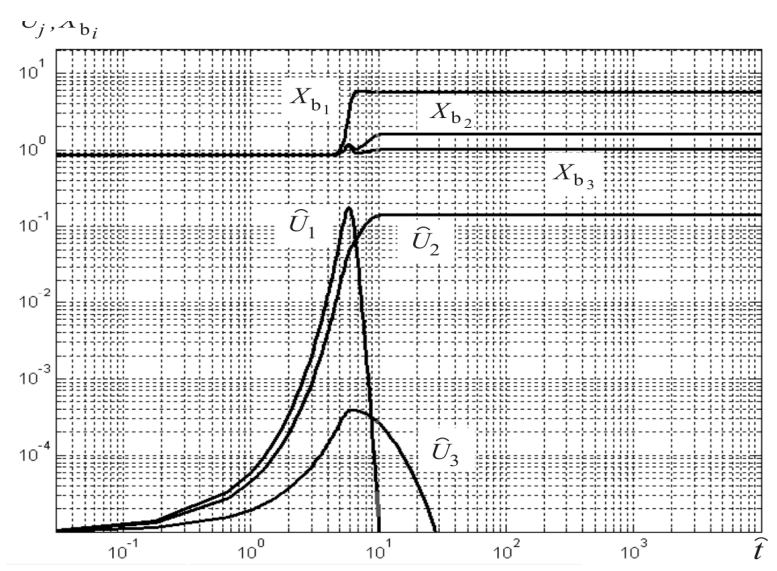

(b)

Figure 2. Distinguished case of appearing competition of oscillations in excitation channels of MPOS: suppressing energetically less active oscillations (a); suppressing energetically more active oscillation by less active one (b).

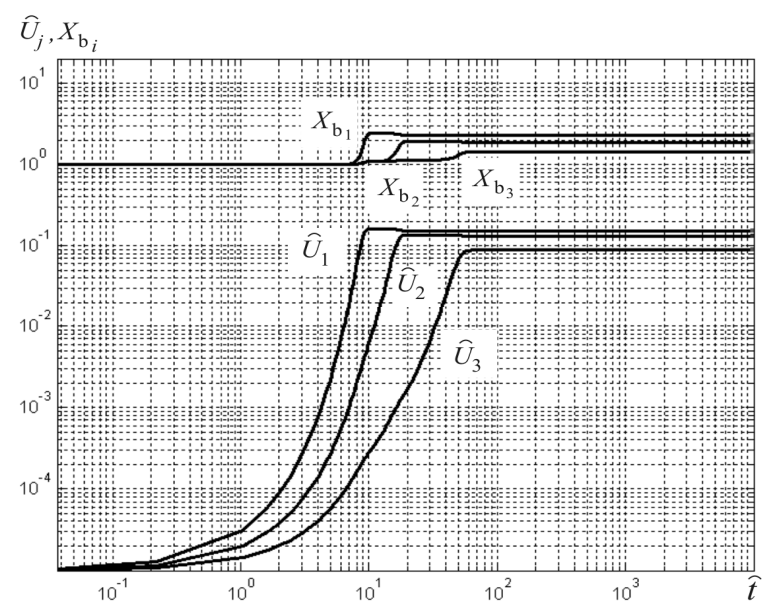

(a) 


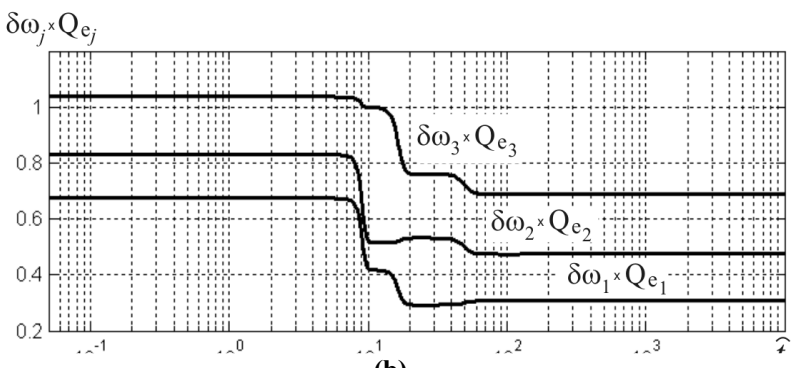

(b)

Figure 3. Stable three-frequency generation mode: setting oscillation amplitudes and auto-bias voltages (a); initial run-out of oscillation frequencies, caused by amplitude and phase conversion in excitation channels (b).

It's obvious that high competition in excitation channels $\left(\gamma_{j i}=-6 \mathrm{~dB}\right)$ can bring to situations when oscillations with low energetic activity (oscillation $\hat{U}_{3}$, fig. 2, a, b) can be suppressed by more active oscillations (with high regeneration coefficient $K_{j}^{r e g}$ ). At the same time the overly high increasing regeneration parameter $K_{j}^{r e g}$ can cause a breakdown of more energetically active oscillations (oscillation $\widehat{U}_{1}$, fig. 2, b) in excitation channels of MPOS. Reducing competition of oscillations due to increasing selective properties of FC circuits down to $\gamma_{j i}=-12 \mathrm{~dB}$ provides stable three-frequency mode of oscillation (fig. 3, a) even when regeneration coefficient values $K_{1}^{\text {reg }}=1.25 K_{2}^{\text {reg }}=1.5 K_{3}^{\text {reg }}$ are growing significantly [23].

In this case it assumes using the term of low or high active oscillation (oscillations) in relation to the other oscillations of MPOS, which, being energetically all-sufficient in one-frequency mode, may fade on transiting to multi-frequency mode of oscillations in the certain conditions.

Figure 4, a represents approximated relationships of oscillation stabilizing time $\tau_{\text {stat }}=\max _{1 \leq j \leq m} \tau_{\text {stat }_{j}}$ at various partial coefficients of FBC. It also has bifurcation curve that determines a range of stable MOM. Evidently, at high values of variations $\chi=\max _{1 \leq j \leq m} K_{j}^{r e g} / \min _{1 \leq j \leq m} K_{j}^{r e g}=\max _{1 \leq j \leq m} R_{\mathrm{e}_{j}} / \min _{1 \leq j \leq m} R_{\mathrm{e}_{j}}$ of regeneration coefficients the oscillation stabilizing time $\tau_{\text {stat }}$ is significantly increasing (by an order and more!). Compensation of this events when having the great scatter of equivalent resistances $R_{\mathrm{e}_{j}}$ is possible by selecting appropriate coefficients $K_{j j}$ of partial FBC (increasing energy of oscillations), what it's not only improving dynamic properties of MPOS, but also brings to extending range of stable MOM (fig. 3, a).

The quite important characteristic of MPOS dynamics is group run-out of oscillation frequencies $\delta \omega_{j} Q_{e_{j}}$, which is defined according to (17). The modeling results indicate that amplitude-phase conversion slightly exhibits for energeti- cally "weak" oscillations (fig. 3, b). At joint control of oscillation amplitude and voltage of auto-bias the relative run-out of frequencies $\delta \omega_{j}=\left(\omega_{q_{j}}\right)^{-1} \cdot \Delta \omega_{j}(\hat{t})=$ $=\left(\omega_{q_{j}}\right)^{-1} \cdot \frac{d \varphi_{j}}{d \hat{t}}$ may reach values from $10^{-8}$ to $10^{-6}$, and significantly reduce on increasing coefficients $K_{j j}$ of partial circuits of FC (fig. 4,b).

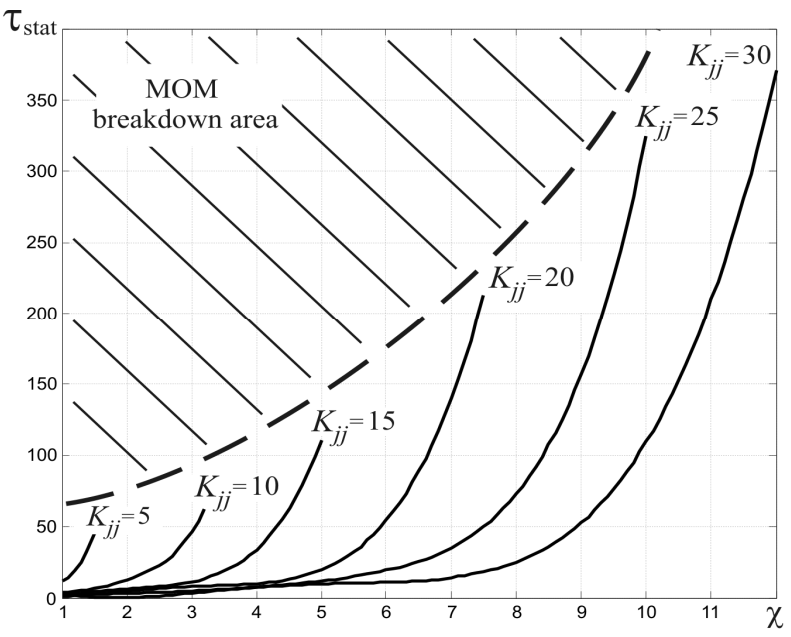

(a)

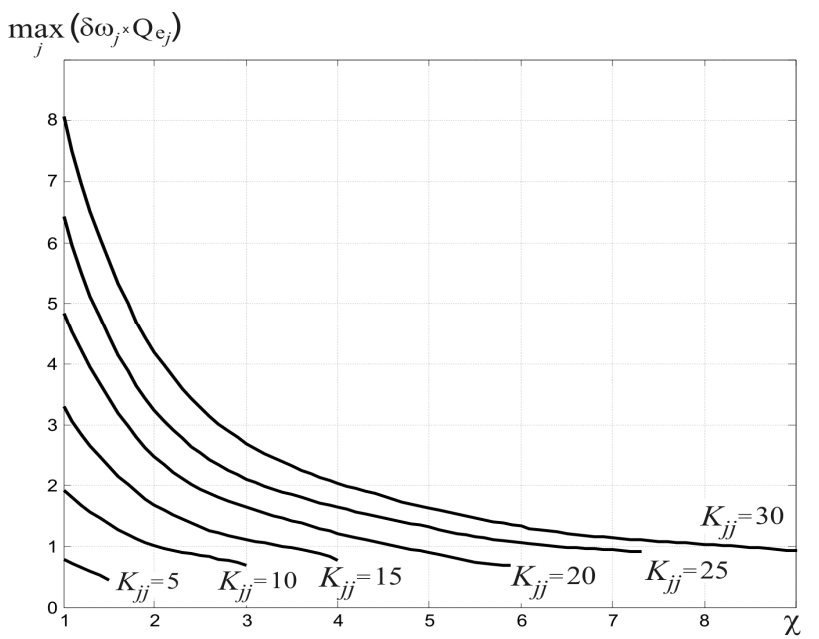

(b)

Figure 4. Dependences of normalized oscillation stabilizing time (a) and initial frequency run-out on MPOS parameters (b).

\section{Conclusion}

The paper proposes the mathematical model of multi-frequency piezoresonance oscillation system - PRD core, which enables to broaden the functional possibilities of multi-frequency - algorithmic methods to provide the invariance of piezoresonance devices. On basis of MPOS structure (fig. 1), which is realized on principles of building filter schemes, the generic truncated amplitude and phase equations (10)-(12) of system movements are derived. The use of generic equations allows removing restrictions on the quantity of generated frequencies while analyzing the 
MPOS dynamics.

On the example of three-frequency oscillation mode the numeral analysis of equations of MPOS movement is done, and the margins of acceptable system parameters are defined, which provide the stable multi-frequency oscillation mode. The character cases of competitiveness of oscillations in excitation channels are presented (fig. 2,3). It is demonstrated that because of high competitiveness of oscillations not only suppression of energetically less active oscillations, but under certain conditions the suppression of energetically more active oscillation by less active one is possible. As the same time, increasing selective properties of FBC chains provides constant three-frequency oscillation mode even under considerably different meanings of regeneration coefficients $\left(K_{1}^{\text {reg }}=1.25 K_{2}^{\text {reg }}=1.5 K_{3}^{\text {reg }}\right)$. This enables not only to manage each separate oscillation mode of quartz resonator, but also change the quantity of simultaneously generated frequencies.

Numerical analysis of equation of movement MPOS demonstrated that under large dispersions of coefficients of regeneration the group time of oscillation determination increases substantially (by order and more!). Compensation of these effects under large dispersion of equivalent resistances is possible by choosing appropriate coefficients of partial FC (by increasing the oscillation power), which not only improves the dynamic properties of MPOS, but also leads to widening the margins of stable MOM (fig. 4).

Rather important characteristic of MPOS is group frequency overshoot $\delta \omega_{j}=\left(\omega_{q_{j}}\right)^{-1} \cdot \Delta \omega_{j}(\hat{t})$. The results of modeling show that amplitude-phase conversion develops little for energetically less active oscillations. Besides minimization of time of MPOS developing oscillation can be provided by means of balancing activeness of generated oscillations (symmetric mode) under the condition of compromise between amplitudes of oscillations (sustainability) and initial frequency overshoot (stability).

Received results demonstrate that the use of developed mathematical model of MPOS, which contains inherently truncated differential equations for amplitude, phases and auto-bias voltageas predictive standard model of PRD core, allows to form a new approach to building piezoresonance units with controlled dynamics [27-30], represented in the form of adaptive controlled systems with predictive standard model and develop on their basis a new class of invariant to destabilizing disturbing PRD factors. Inherently such approach has the principle of using natural duplication in multi-frequency basis of PRD core - multi-frequency piezoresonance oscillation system, which enables not only to synthesize the system with current identification of disturbing factors on basis of theory of invariance, but also carry out the adaptation of PRD in accordance with their effects.

\section{References}

[1] Barzhin V. (1972). Multiwave quartz crystal resonator temperature sensor / V. Barzhin, A. Zelensky, F. Kolpakov [etc.] // Electronic Engineering. Ser. 10, Radiokomponenty. 1972. - Issue. I, Moscow, Russia - pp. 54-57.

[2] Rosati V. (1983). State of the art in crystal oscillators, present and future / V. Rosati [etc.] // MILCOM-83. Proc. IEEE Milit., - 1983. - V.2, - P. 386 - 390.

[3] Petrov B. Selected Works / B. Petrov, v.1. - Moscow: Nauka, 1983. - 286 p.

[4] Kolpakov F. Using multiparameter sensitivity piezoelectric resonators in measuring devices / F. Kolpakov // Electronic Engineering. Ser. Radiodetaly and radiokomponenty. - 1985.Issue. 2 (59), Moscow, Russia - pp. 62-66.

[5] Mourey M. (1985). New design of a dual-mode Quartz crystal oscillator / M. Mourey, J. Vaterkovski // Electronics Letters.-1985.-V.21.-№ 5. - pp. $184-186$.

[6] Benjaminson A., Stallings S. (1989). A Microcomputer Compensated Crystal Oscillator using a Dual-Mode Resonator // Proc. of the 43rd Annual Symposium on Frequency Control, 1989, pp. 20-26.

[7] Schodowski S. (1989). Resonator self-temperature-sensing using a dual-harmonic-mode crystal oscillator // Proc. of the 43rd Annual Symposium on Frequency Control, - 1989, pp. 2-7.

[8] Filler R., Vig J. (1989). Resonators for the Microcomputer Compensated Crystal Oscillator // Proc. of the 43rd Annual Symposium on Frequency Control, 1989, pp. 8-15.

[9] Besson R. (1993). A Dual-Mode Thickness-Shear Quartz Pressure Sensor / R. Besson, J. Boy, B. Glotin [etc.] // IEEE Transaction on Ultrasonics, Ferroelectrics, and Frequency Control, Vol. 40, No. 5, pp. 584-591, Sept. 1993.

[10] Kosykh A., Abramson I., Bagaev V. (1994). Dual-mode Crystal Oscillators With Resonators excited on Band C- modes // Proc. of the 48th Annual Symposium on Frequency Control, 1994, pp. 578-586.

[11] Prokopenko V. (1994). Dual Frequency crystal oscillator / V. Prokopenko // Radiotehnika, Vol. 11, ISSN 0033-8486, Moscow, Russia, pp. 24 - 26.

[12] Shmaly Y. (1995). The modulation method of the precision quartz-crystal oscillators and standards frequency stabilization // Proc. of the 49th Annual Symposium on Frequency Control, 1995, pp. 579-589.

[13] Tsarapkin D. (2000). Novel technique for dual-mode quartz oscillators // Frequency Control Symposium and Exhibition, 2000. Proceedings of the 2000 IEEE/EIA, 2000, pp. $425-$ 429.

[14] Oita T., Fukuda M., Nakamura A. [etc.]. (2004). Dual Mode SC-Cut Crystal Oscillator // 2004 IEEE International Ultrasonics, Ferroelectrics, and Frequency Control Joint 50th Anniversary Conference, 2004, pp.436-442.

[15] Stofanik V., Balaz I., Minarik M. (2007). Dual-Mode Crystal Oscillator with Simultaneous Excitation of Two Overtones in a Stress Compensated Quartz Resonator // Proceedings of the joint 2007 European Frequency and Time Forum and the 2007 IEEE International Frequency Control Symposium, 2007, pp. 227-229. 
[16] Kolpakov F., Pidchenko S. (2011). Theory and fundamentals implementation of invariant piezoresonance systems // Published by National Aerospace University (KhAI), ISBN 978-966-662-222-1, Kharkov, Ukraine.

[17] Zelensky A., Pidchenko S. (2011). Principles of invariant piezoresonance oscillatory systems // 4th International Radio Electronic Forum (IREF'2011): Proceedings of the International Conference ICTST'2011, October 18-21, Kharkov, Ukraine, Vol. 2, pp. 32-35.

[18] Kolpakov F., Pidchenko S., Taranchuk A. (2008). Invariant piezoresonance oscillatory systems // Measuring and Computing Devices in Technological Processes, Vol. 1, Khmelnitsky national university, ISSN 2219-9365, Khmelnitsky, Ukraine, pp. 174-190.

[19] Pidchenko S., Taranchuk A. (2004). Identification of the thermal state of the crystal at the stage of oscillation // Radioelectronic and computer system, Vol. 3, National Aerospace University (KhAI), ISSN 1814-4225, Kharkov, Ukraine, pp. 36-42.

[20] Taranchuk A., Pidchenko S. (2005). Modelling of thermal processes in the piezoresonance sensors with modulated interelectrode gap // Khmelnitsky State University's bulletin, Vol. 1, Khmelnitsky national university, ISBN 978-966-330-114-3, Khmelnitsky, Ukraine, pp. 218-222.

[21] Pidchenko S. Taranchuk A., Stetsyuk V. (2011). Mathematical modeling force-frequency characteristics of the quartz resonators // Radioelectronic and computer system, Vol. 2, National Aerospace University (KhAI), ISSN 1814-4225, Kharkov, Ukraine, pp. 27 - 31.

[22] Zelensky A., Pidchenko S., Taranchuk A. (2012). Multifrequency core structure of an invariant quartz oscillatory system //11th International Conference on "Modern Problems of Radio Engineering, Telecommunications and Computer Science" (TCSET'2012). Lviv-Slavske, Ukraine, 2012. - P. 125 .

[23] Kolpakov F., Pidchenko S., Hilchenko G. (1997). Features of setting process of the oscillations in the multi-channel multi-frequency crystal oscillator // Radiotehnika, Vol. 12, ISSN 0033-8486, Moscow, Russia, pp. 95 - 98.
[24] Kolpakov F., Pidchenko S., Hilchenko G. (1999). Minimization of settling time of oscillations in multi-channel multi-frequency crystal oscillator // Radiotehnika, Vol. 2, ISSN 0033-8486, Moscow, Russia, pp. 42 - 44.

[25] Pidchenko S., Kolpakov F., Akulinichev A. (2000). Analysis of the characteristics of multi-frequency controlled crystal oscillator // Measuring and Computing Devices in Technological Processes, Vol. 3, Khmelnitsky national university, ISSN 2219-9365, Khmelnitsky, Ukraine, pp. 70-75.

[26] Kolpakov F., Pidchenko S. (1999). Syntheses of manychannel multifrequency quartz crystal oscillators with reduced (shortened) time of adjusting oscillations // Zarubejnaya radioelectronica, Vol. 11, ISSN 0373-2428, Moscow, Russia, pp. 60 - 65.

[27] Taranchuk A., Pidchenko S., Opolska A. (2010). Utilization Features of the Mexanotron for Information Measurement Systems // Modern Problems of Radio Engineering, Telecommunication and Computer Science: Procedings of the Xth International Conference TCSET'2010. February 23-27, 2010. - Lviv-Slavske, Ukraine. - P. 358.

[28] Taranchuk A., Pidchenko S. (2012). Design Methodology to Construct Information Measuring Systems Built on Piezoresonant Mechanotrons with a Modulated Interelectrode Gap// Applied Measurement System. Published by InTech, ISBN 978-953-51-0103-1, Janeza Trdine 9, 51000 Rijeka, Croatia, 2012, pp. 229-258.

[29] Taranchuk A., Pidchenko S., Mishan V. (2012). Frequency-compensated piezoresonance oscillator system with external MEMS control V. // 11th International Conference on "Modern Problems of Radio Engineering, Telecommunications and Computer Science" (TCSET'2012). Lviv-Slavske, Ukraine, 2012. - P. 458.

[30] Kolpakov F., Slavinsky S. (2004). The sphygmography measuring converter "pressure-frequency" dynamic characteristics examination // Measurement Science Review, Vol. 4, Sect. 2, pp. 52-58. 